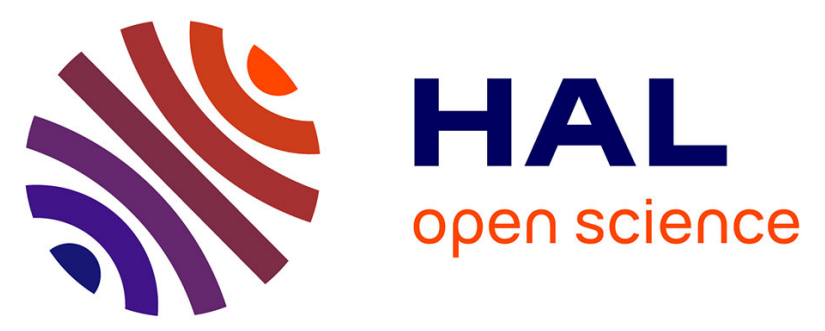

\title{
Hemodynamic responses to visual cues during attentive listening in autonomous versus manual simulated driving: A pilot study
}

Antonio R. Hidalgo-Munoz, Christophe Jallais, Myriam Evennou, Daniel Ndiaye, Fabien Moreau, Maud Ranchet, Romain Derollepot, Alexandra Fort

\section{To cite this version:}

Antonio R. Hidalgo-Munoz, Christophe Jallais, Myriam Evennou, Daniel Ndiaye, Fabien Moreau, et al.. Hemodynamic responses to visual cues during attentive listening in autonomous versus manual simulated driving: A pilot study. Brain and Cognition, 2019, 135, 8 p. 10.1016/j.bandc.2019.103583 . hal-02271104

\section{HAL Id: hal-02271104 \\ https://hal.science/hal-02271104}

Submitted on 26 Aug 2019

HAL is a multi-disciplinary open access archive for the deposit and dissemination of scientific research documents, whether they are published or not. The documents may come from teaching and research institutions in France or abroad, or from public or private research centers.
L'archive ouverte pluridisciplinaire HAL, est destinée au dépôt et à la diffusion de documents scientifiques de niveau recherche, publiés ou non, émanant des établissements d'enseignement et de recherche français ou étrangers, des laboratoires publics ou privés. 
Post-print : HIDALGO-MUNOZ, Antonio R., JALLAIS, Christophe, EVENNOU, Myriam, NDIAYE, Daniel, MOREAU, Fabien, RANCHET, Maud, DEROLLEPOT, Romain, FORT, Alexandra, 2019, Hemodynamic responses to visual cues during attentive listening in autonomous versus manual simulated driving: A pilot study, Brain and Cognition, 135, Elsevier, 8 p, DOI: 10.1016/ j.bandc.2019.103583

\title{
Hemodynamic responses to visual cues during attentive listening in autonomous versus manual simulated driving: A pilot study
}

Antonio R. Hidalgo-Muñoz ${ }^{* 1}$, Christophe Jallais ${ }^{1}$, Myriam Evennou ${ }^{1}$, Daniel Ndiaye ${ }^{2}$, Fabien Moreau $^{1}$, Maud Ranchet ${ }^{1}$, Romain Derollepot ${ }^{1}$, Alexandra Fort ${ }^{1}$

${ }^{1}$ Université de Lyon, IFSTTAR, TS2, LESCOT, F-69675, Lyon, France

${ }^{2}$ Université Paris-Est, IFSTTAR, COSYS, LEPSIS, F-77447, Marne-la-Vallée, France

\begin{abstract}
Emerging automation technologies could have a strong impact on the allocation of drivers' attentional resources. The first objective of this pilot study is to investigate the hemodynamic responses evoked to relevant visual stimuli in manual and autonomous driving. The second aim is to examine how the inclusion of a secondary task (attentive listening to a broadcast) modulates these hemodynamic responses in both driving situations. Frontal, temporo-parietal and occipital activations were recorded using a functional Near-InfraRed Spectroscopy (fNIRS) system. Event-related analysis was used to determine whether visual cue processing (specifically, the lighting of a lead vehicle's brake-lights) could induce different brain responses depending on the driving mode and on the presence or absence of a competing task. Mind-wandering as reported by the participants was more pronounced during autonomous compared to manual driving. Our results showed an increase in the OxyHb concentration in the right temporo-parietal and occipital areas during manual compared to autonomous driving, suggesting greater allocation of attentional resources for processing visual cues in the first condition. Finally, an event-related decrease in right frontal activity during autonomous driving when listening was observed, suggesting that attentional resources were more focused on the secondary task than on monitoring the driving scene.
\end{abstract}

Keywords: attention, auditory stimuli, distraction, driving simulator, fNIRS, 


\section{Introduction}

Manual driving is a demanding cognitive and physical task, the performance of which could be affected by distraction and inattention (Fort et al., 2010; Lemercier et al., 2014). Driver distraction can be caused by everyday, concurrent tasks involving interactions with an object (Caird et al., 2018) or by external stimuli unrelated to the driving context. Concerning inattention, it can be defined as a decrease in attentional resources allocated to driving because of thoughts unrelated to the driving task, either voluntary thoughts directed towards a goal (e.g., problem solving), ruminations (Jallais, Gaubaude and Paire-Ficout, 2014) or mindwandering (Smallwood and Schooler, 2015; Baldwin et al., 2017).

New automation technologies and advanced driver-assistance systems in cars can facilitate distraction and inattention. As they carry out several tasks, such as speed regulation or collision risk warning, these systems may change the division of attentional resources by reducing the attentional demand of the driving task and thus increasing the driver's proneness to distraction (Xu et al., 2017) or inattention. Given the safety consequences of distraction and inattention in both manual (see Galéra et al. 2012) and autonomous driving, when the driver is expected to take over (see Navarro, 2018), it is crucial to understand how distraction may affect information processing in both conditions. To be able to assess how distraction and inattention can modulate information processing during autonomous driving, that is when no driving performance is required, the use of neuroimaging techniques is of great interest.

As the driving task involves a large range of perceptual and cognitive processes (e.g., perception, selection, anticipation, planning, decision-making and motor coordination), this activity recruits a large cerebral network. In a meta-analysis of functional magnetic resonance imaging (fMRI) studies, Navarro et al. (2018) identified what they call a "common cardriving circuit" composed of the cerebellum (motor coordination), the bilateral extra-striate cortex (visual selective attention and visual processing), the right middle temporal gyrus (visual motion processing), the precuneus (visuospatial information processing), the left anterior part of the insula (high level cognitive control and attentional processes), the right posterior cingulate gyrus (topographical memory), the right dorsomedial part of the thalamus (corticocortical interactions) and motor areas.

Distraction and inattention have been examined using neuroimaging techniques. Allen et al., (2013), using blood-oxygen-level-dependent (BOLD) activity, found that medial prefrontal cortex (mPFC) activity increased when thoughts unrelated to the task increased, whilst dorsal region activity decreased when the individual engaged with the task. In the driving context, Just et al. (2008) showed, also using fMRI, that listening to statements and judging their veracity decreases activity in a parietal lobe region involved in spatial processing compared to a driving without listening to the statements. Similarly, using magnetoencephalography (MEG), Fort el al., (2010) examined how a distractive listening task affects neuronal processing of driving-relevant visual information (traffic light or direction). They observed that divided attention entails a reduction in top-down attention for visual processing. This reduction results in decreased activity in the dorso-lateral prefrontal cortex linked to selection and top-down attention (Corbetta \& Shulman, 2002) and in the occipital areas linked to perceptual processing. In addition, these authors observed an increase in activity in the posterior parietal region involved in attentional shifting (Vandenberghe et al. 
2001). Despite their interest, both techniques (fMRI and MEG) require that participants keep head movements to a minimum. Moreover, fMRI requires participants to lie down. These constraints limit immersion in simulated driving.

The recent deployment of functional near infrared spectroscopy (fNIRS) offers a new possibility for investigating brain responses in more naturalistic settings such as driving (Unni, Ihme, Jipp and Rieger, 2017). However, few studies have compared cognitive workload during manual and autonomous driving using fNIRS. Sibi, Ayaz, Kuhns, Sirkin, and $\mathrm{Ju}$, (2016) aimed to determine the cognitive workload variation in drivers utilizing vehicles with different levels of automation. They found that autonomous driving decreases frontal activation compared to manual driving, suggesting a lower cognitive load during autonomous driving. This reduced load is a concern as it has been suggested that intermediate levels of automation that require unexpected take-over can unsettle drivers if they are not ready to resume control (Arakawa, Hibi \& Fujishiro 2018).

Although the purpose of the studies on the topics mentioned above was to evaluate brain activation while driving and executing secondary tasks, the event-related paradigm to investigate hemodynamic responses to visual cues has been scarcely considered. On the other hand, the influence of attentive listening as a distractive task in manual and autonomous driving has not been studied yet. Most studies using fNIRS whilst driving investigated the prefrontal cortex (Liu et al., 2016) even though driving involves a large neural network as reported above. Therefore, the objective of this study is twofold. The first aim is to investigate the hemodynamic responses evoked to relevant visual stimuli in manual and autonomous driving. The second aim is to examine how the inclusion of a secondary task (attentive listening to a broadcast) modulates these hemodynamic responses in both driving situations.

It is hypothesized that autonomous driving allows greater mind-wandering than manual driving, which is more attentionally demanding. Thus, mind-wandering should decrease the available attentional resources to process visual cues during autonomous driving compared to manual driving. When performing a secondary task during autonomous driving, although mind-wandering should decrease, the attention would be oriented to this secondary task (distractive task) and contribute to decreasing the attentional resources to process visual cues. Hence, a difference between mind-wandering and conducting a secondary task during autonomous driving should be reflected in increased activity in the brain areas underlying processing of the secondary task and decreased evoked response amplitude in the prefrontal cortex (Fort et al. 2010; Allen et al. 2013).

To test these hypotheses, we designed an experiment using a car simulator while recording event-related hemodynamic activity using fNIRS. Since frontal, temporo-parietal and occipital areas are recruited during the driving task and for multitasking, cortical activity was measured in these regions.

\section{Materials and method}

\subsection{Participants}

A total of 12 healthy, French-speaking volunteers $20-43$ years of age (mean $=29.3 \pm$ 7.6, 6 males, one left-handed) from The French Institute of Science and Technology for 
Transport, Development and Networks (IFSTTAR) were recruited for the experiment. All participants had normal auditory acuity, normal or corrected-to-normal vision and no history of a neurological or psychological disorder (according to self-report). A valid driving license for at least 3 years was required $(10.8 \pm 8$ years). This study was approved by the French ethical committee (Comité de protection des personnes Sud-Est1, reference number: 2018A01202-53). All subjects gave written informed consent in accordance with the Declaration of Helsinki.

\subsection{Simulation and driving tasks}

The driving simulator was equipped with a steering wheel, a turning indicator, an accelerator and a brake pedal. An interactive visual scene of a suburban driving environment was projected onto three screens covering an angle of $150^{\circ}$. Participants were comfortably seated in a car seat approximately $80 \mathrm{~cm}$ from the central screen (24") and asked to avoid abrupt head movements. Two different driving activities were required:

- For the manual driving sessions, the participant was instructed to drive in a suburban environment and follow a car that joined the lane. There were no oncoming vehicles, and overtaking was not allowed. The participant had to try and keep a speed of $70 \mathrm{~km} / \mathrm{h}$ and brake when they saw brake lights from the car in front. The scenario finished with an oral message to park.

- For the autonomous driving sessions, the participant was asked to start driving the vehicle and delegate control to the autonomous system by taking their hands off the steering wheel and their feet off the pedals as soon as a sound alert was emitted to trigger autonomous driving. During autonomous driving, the vehicle drove automatically, and the participant was asked only to pay attention to the driving environment and the brake lights from the lead car. No driving action from the participant was needed for autonomous driving. The vehicle drove and braked in an autonomous way, uninterrupted. The scenario finished with an oral message to park manually.

\subsection{Auditory stimuli}

The auditory stimuli consisted of 8 radio broadcasts 2 minutes in length extracted from the Internet (creative commons license) that dealt with different topics, such as history, literature and science. In each broadcast, a main narrator (gender parity among the broadcasts) talks about a particular topic in an informal style. The emotional content of the stimulus was assessed by an adaptation of the Geneva Emotion Wheel (Scherer, 2005), where the intensity of different feelings (happiness, energy, serenity, compassion, anger, boredom, fear, anxiety and sadness) were self-rated by the participants. In addition, the participants' task engagement was evaluated using a Likert scale (from 0: no interest/boring to 5: very interesting) to check the possible influence of boring or interesting content on brain activation (Horrey et al., 2017).

To ensure that the participant listened attentively to the broadcasts, they were informed that they had to answer three questions about the content of each broadcast when it ended. The responses were scored with $0,0.5$ or 1 point (always evaluated by the same experimenter) 
according to their accuracy. The broadcasts were randomly selected for each condition to avoid repetition.

\subsection{Mind-wandering assessment}

The tendency for mind-wandering was assessed at the beginning of the experiment by the French version of the Mindful Attention Awareness Scale (MAAS) (Jermann et al., 2009). The MAAS is a 15-item scale designed to assess a core characteristic of dispositional mindfulness, namely, open or receptive awareness of and attention to what is taking place in the present (Brown \& Ryan, 2003).

The proportion of attention allocated to the driving task and the proportion of thoughts unrelated to the tasks (driving and listening) considered as mind-wandering were estimated by each participant for each driving session. The participants were instructed to indicate the percentage of thoughts related to driving, to the broadcast (for dual-task condition) and "other thoughts".

\subsection{Experimental design}

After the lead vehicle joined the lane, ten braking actions had to be executed for each driving session. The lead vehicle braked with a random inter-stimulus duration between 7 and $10 \mathrm{~s}$ and the participant had to brake as quick as possible to avoid to reach the car. Manual and autonomous driving activities (described in section 2.2), were carried out with and without listening to broadcasts and were considered the dual task and single task, respectively.

Two sessions 2 minutes in length for each factor combination were presented (manual or autonomous conditions for the driving factor and dual task or single task for the listening factor), i.e., 2 sessions $\times 2$ factors $\times 2$ levels $=8$ driving sessions (see an example for a participant in Fig. 1). The order in which the driving conditions were presented was counterbalanced. In the case of dual-task conditions, the participant had to answer orally the three questions about the broadcast content just after it ended. Then, after each session, the participant answered the question about the proportion of thoughts related to each task or unrelated to them (mind-wandering) in approximately 15 seconds in writing by indicating the percentage (\%). Then, the participant had 30 seconds to recover a resting state. After the first run (consisting in 4 driving sessions), there was a break if participant needed it. 


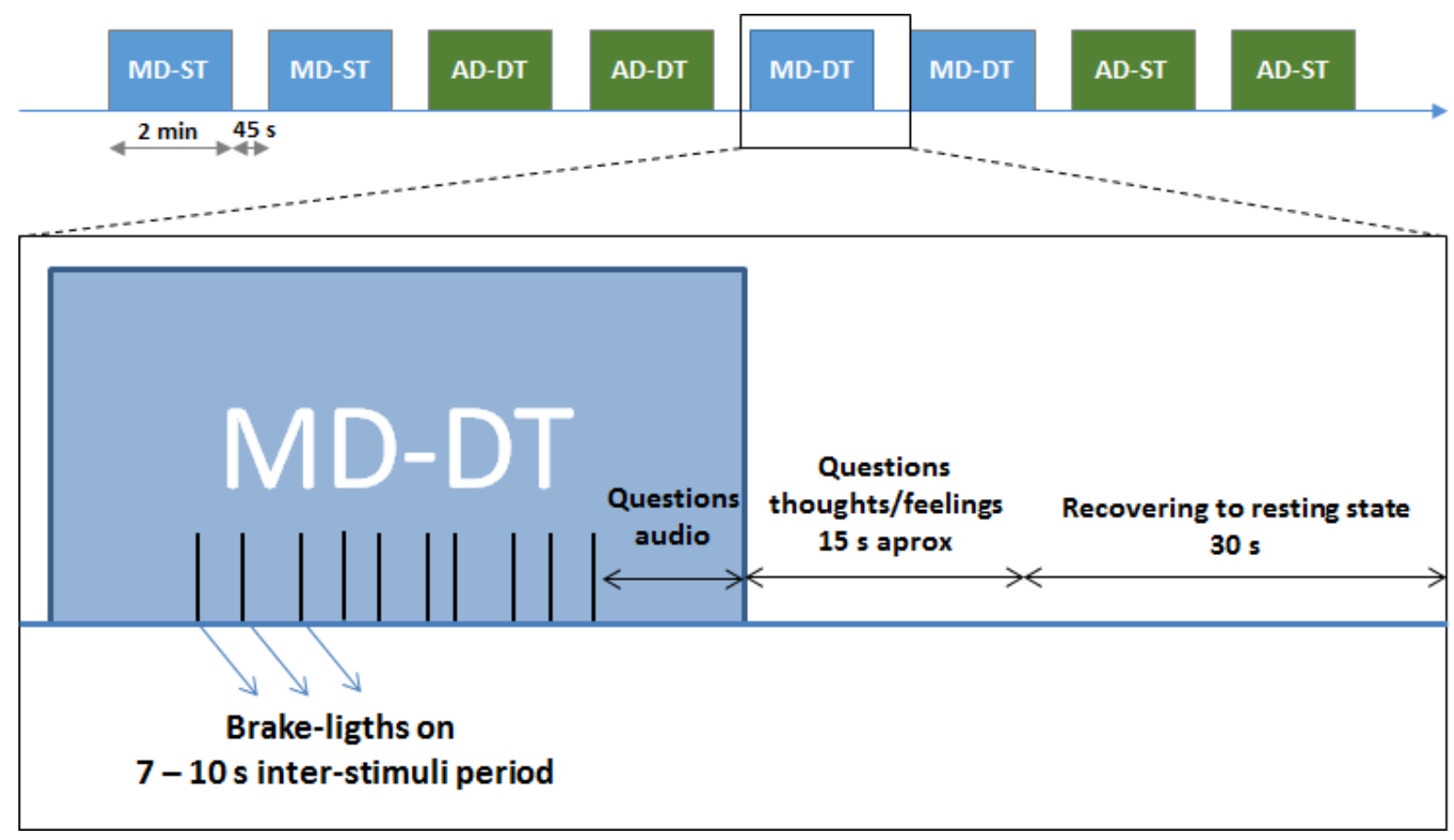

Figure 1: Example of driving session order for one participant. In each session of 2 minutes, 10 braking action are expected. $\mathrm{MD}=$ manual driving; $\mathrm{AD}=$ autonomous driving; $\mathrm{ST}=$ single task; $\mathrm{DT}=$ dual task.

\section{6. fNIRS recording}

A NIRScout device (NIRx Medical Technologies) was used for fNIRS data acquisition. The system has 16 sources and 24 detectors ( 22 of them included in this study) that receive wavelengths of 760 and $850 \mathrm{~nm}$ recorded at $3.9 \mathrm{~Hz}$ to compute OxyHb and DeoxyHb concentrations, respectively. Optode placement choice was based on the recommendations of Morais, Balardin and Sato (2018) to cover auditory, frontal and occipital areas and constrained to keep an appropriate source-detector distance of $3 \mathrm{~cm}$, resulting in 41 channels as depicted in Fig. 2.

A calibration was performed for each participant to check each optode's signal quality to guarantee an acceptable gain level. Dark noise analysis assured a low luminosity pollution for the measures.

\section{7. fNIRS signal processing}

The fNIRS signals were analyzed using NirsLAB toolbox version 2017.6 (Xu, Graber and Barbour, 2014). A band pass filter of $0.01-0.5 \mathrm{~Hz}$ was applied to discard cardiac activity, respiratory signals and high frequency noise. Spike artifacts and discontinuities were removed with STD thresholds of 3 and 5, respectively. Averaged oxygenation concentrations were computed using the modified Beer-Lambert law for each epoch (Villringer, Planck, Hock, Schleinkofer \& Dirnagl, 1993). 


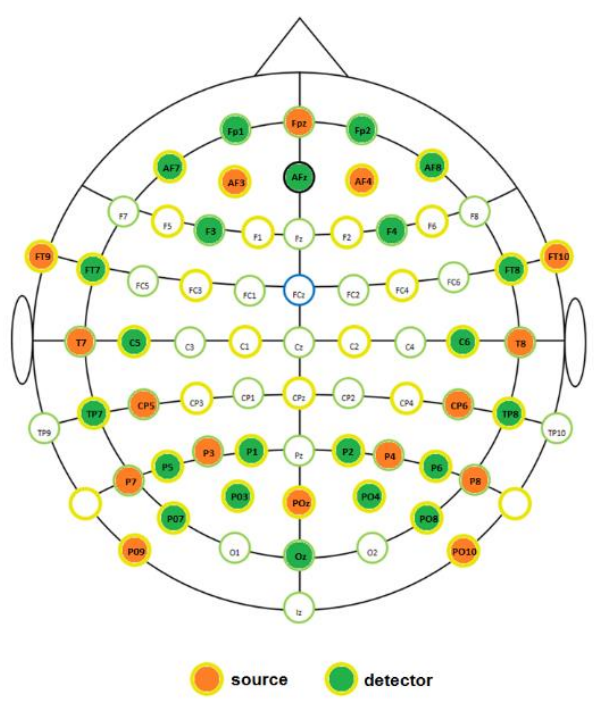

(a)

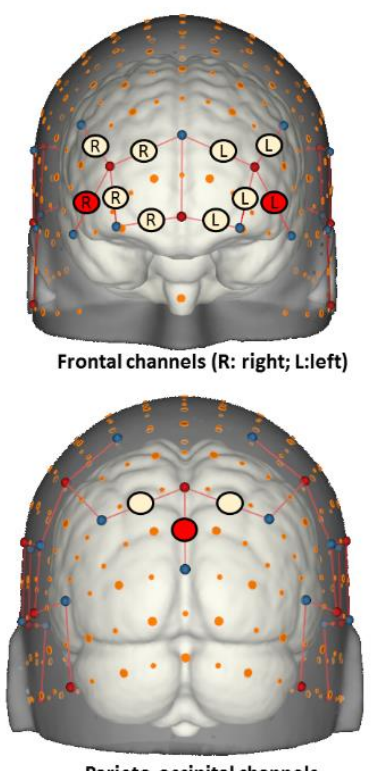

Parieto-occipital channels

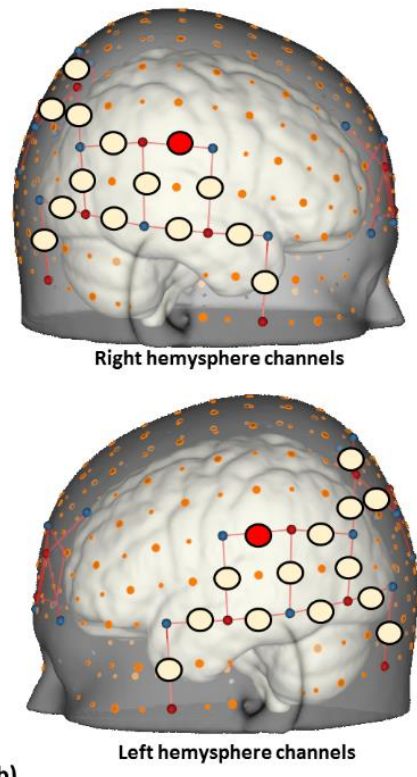

(b)

Figure 2: (a) Probe setup (optode positions) according to the 10-20 system. (b) Channel clustering for brain area analysis. Of note, frontal channels were separated into two regions according to laterality. Red channels were utilized for visual representation of the grand average signals (see Figs. 4 and 5).

The event related responses were computed within $7 \mathrm{~s}$ segments after the stimulus (brake lights), and a maximum of 30 points were sampled to define the curves. The application of a jitter (variable inter-stimuli interval duration) prevented spurious periodic artefacts in the event-related responses. The approach to study the evoked hemodynamic responses can be considered a rapid event-related study where the inter-stimuli intervals may be shorter than the response elicited (Amaro Jr. \& Barker, 2006).

Given the short segments in the study, the mean signal value was the most representative parameter for information linked to brain area activation compared to the maximal peak value.

An exploratory analysis (paired Student's t-tests) was performed to determine the most relevant channels. However, given that current fNIRS systems do not let an adequate spatial resolution (a distance about $3 \mathrm{~cm}$ between source and detector is required) to study constricted brain regions, an exhaustive brain topological study was out of the scope of this paper. Therefore, to avoid the influence of individual differences in placement accuracy and to reduce the artefacts linked to basal blood flow, the channels were grouped in different clusters and the averaged signals computed for further statistical analysis.

\subsection{Statistical analysis}

Sphericity was first checked for the OxyHb and DeoxyHb values by means of Mauchly's test. Greenhouse-Geisser correction was applied if the sphericity assumption was not met.

A two-way analysis of variance (ANOVA $2 \times 2$ ), the first factor representing the driving activity (manual and autonomous) and the second one referring to the concurrent listening task (single and dual task), was performed for each variable for every group of 
channels according to the scheme showed in Fig. 1. Post-hoc analyses (LSD correction) were carried out to disentangle significant interactions.

All statistical analyses were carried out using SPSS 25.0 software.

\section{Results}

\subsection{Auditory attention assessment}

Listening attention was assessed by answers to three questions for each broadcast. Given that there are two dual task sessions for each driving condition (see Sec. 2.5), the maximal score was 6 for each condition. There were no significant differences in attention during autonomous $(4.3 \pm 1.3$ correct answers $)$ and manual driving $(4 \pm 1.4)$ conditions $(\mathrm{p}=$ $.61)$. The correlation between attention scores in each condition was not significant $(r=.01, p$ $=.8)$.

\subsection{Mind-wandering scores}

Figure 3 shows the percentage of thoughts related to the driving task or driving environment for manual and autonomous driving. The thoughts linked to the broadcast content (only for the dual task conditions) and other thoughts considered mind-wandering were also considered. As depicted in Fig. 3, attention payed to the driving context was higher for manual driving, even if the participant was instructed to pay attention to the lead vehicle during autonomous driving. As expected, listening to a broadcast decreased the mindwandering linked to other thoughts.

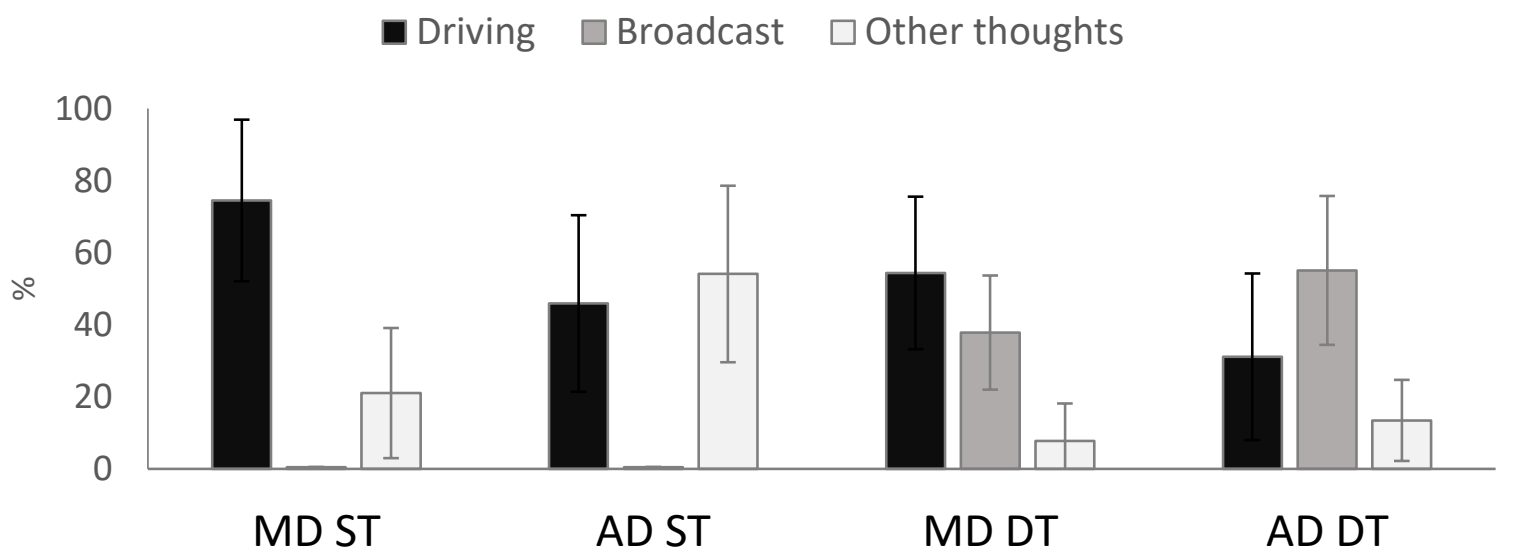

Figure 3: Percentage of thoughts related and unrelated to the tasks in each of the 4 conditions. MD: Manual Driving; AD: Autonomous driving; ST: single task; DT: dual task.

The averaged MAAS scores of the 12 participants was 3.29 (standard deviation = 1.02), which is similar to the results presented for the French population norm by Jermann et al., 2009. None of the participants was considered outliers according to MAAS results. A significant correlation was found between the MAAS score and performance in the listening task during autonomous driving $(\mathrm{r}=-.671 ; \mathrm{p}=.034)$, indicating that individuals with a higher MAAS score were less accurate in answering questions in the autonomous driving condition. 
Regarding the interest level, no significant main effect $(p=.078)$ was found despite the average score generally being higher for dual task conditions.

\subsection{Brain activation analysis}

\subsubsection{Main effects: driving and listening}

Concerning the $\mathrm{OxyHb}$ mean values, a main effect of driving mode was found in the temporo-parietal area of both hemispheres (left: $\mathrm{F}(1,11)=7.06 ; \mathrm{p}=.022 ; \eta_{\mathrm{p}}{ }^{2}=0.391$ and right: $\left.\mathrm{F}(1,11)=8.44 ; \mathrm{p}=.014 ; \mathrm{n}_{\mathrm{p}}^{2}=0.434\right)$ that showed higher activation for manual driving than for autonomous driving.

Regarding the DeoxyHb mean values, a main effect of driving mode was found for the right frontal area $\left(\mathrm{F}(1,11)=8.63 ; \mathrm{p}=.013 ; \eta_{\mathrm{p}}{ }^{2}=0.440\right)$ and for the left tempo-parietal region $\left(\mathrm{F}(1,11)=5.61 ; \mathrm{p}=.037 ; \eta_{\mathrm{p}}^{2}=0.338\right)$ that showed, in contrast to OxyHb values, higher concentrations in autonomous driving.

No main effect of listening was found for OxyHb or DeoxyHb.

\subsubsection{Interaction: driving and listening}

An interaction between driving mode and listening was significant for both frontal areas (left: $\mathrm{F}(1,11)=6.91 ; \mathrm{p}=.023 ; \eta_{\mathrm{p}}{ }^{2}=0.386$ and right: $\mathrm{F}(1,11)=8.46 ; \mathrm{p}=.014 ; \eta_{\mathrm{p}}{ }^{2}=$ $0.435)$, the right temporo-parietal area $\left(\mathrm{F}(1,11)=6.46 ; \mathrm{p}=.027 ; \eta_{\mathrm{p}}{ }^{2}=0.370\right)$ and the occipital area $\left(\mathrm{F}(1,11)=6.44 ; \mathrm{p}=.028 ; \mathrm{n}_{\mathrm{p}}^{2}=0.369\right)$.

To disentangle the origin of these interactions, post-hoc analyses were performed, and the results are illustrated in Figures 4, 5 and 6. The mean values of OxyHb and DeoxyHb following the visual cue (illumination of the brake light on the lead vehicle) was computed over 30 points. The results revealed that activation of the right temporo-parietal $(\mathrm{p}=.013)$ (Fig. 4) and occipital ( $\mathrm{p}=.011$ ) (Fig. 5) areas are higher in manual driving compared to autonomous driving in the dual task condition (when the listening task competes). Figures 4 shows an earlier amplitude peak for the occipital region during manual driving (between 3 and $4 \mathrm{~s}$ after stimulus presentation) than for right temporo-parietal regions (between 4 and $5 \mathrm{~s}$ ) as depicted in Fig. 5.

A similar result to this one was found in the right frontal area (Fig. 6) where the activation decreases significantly in autonomous driving in the dual task condition compared to $\mathrm{ST}(\mathrm{p}=.038)$. 


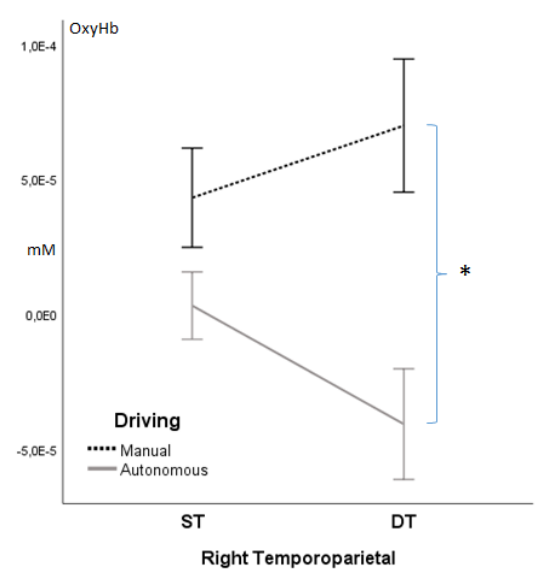

(a)

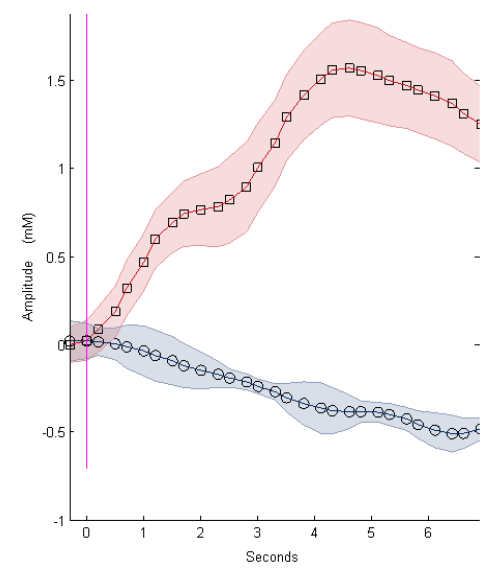

MD-DT

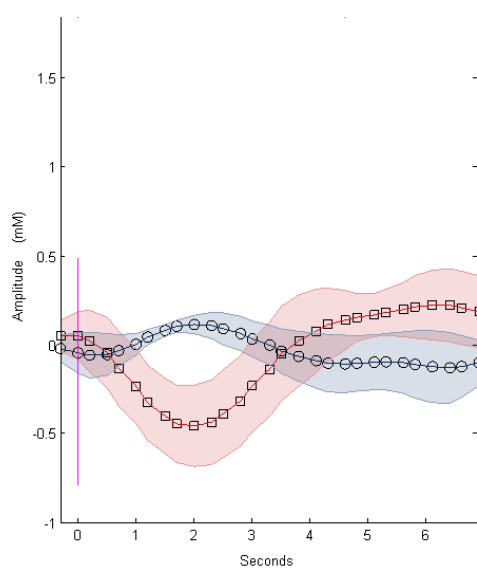

AD-DT

(b)

Figure 4: (a) Interaction between driving mode and listening for OxyHb in the right temporoparietal region. ST: Single task. DT: Dual task (with listening). (b) Grand average (including 12 participants, mean \pm standard error) hemodynamic responses from right temporoparietal region in DT by manual (MD) or autonomous (AD) driving condition, and the mean \pm standard error inter-subject of OxyHb (red lines) and DeoxyHb (blue lines) are represented for the channel between C6 and CP6 (amplitude $\left.(\mathrm{mM}) \times 10^{-4}\right)$.

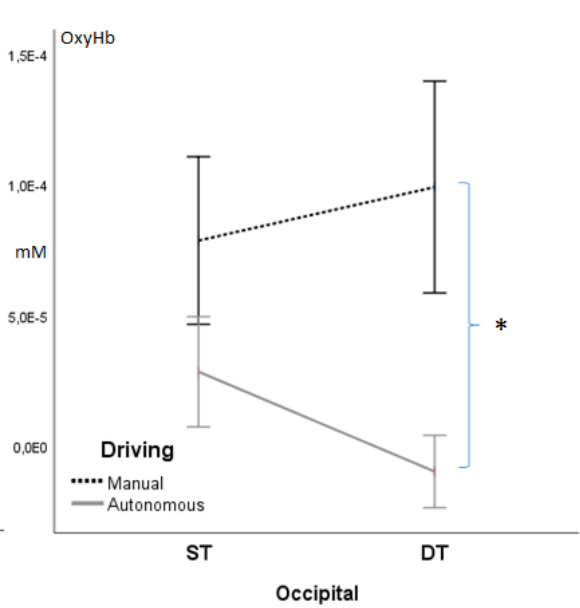

(a)

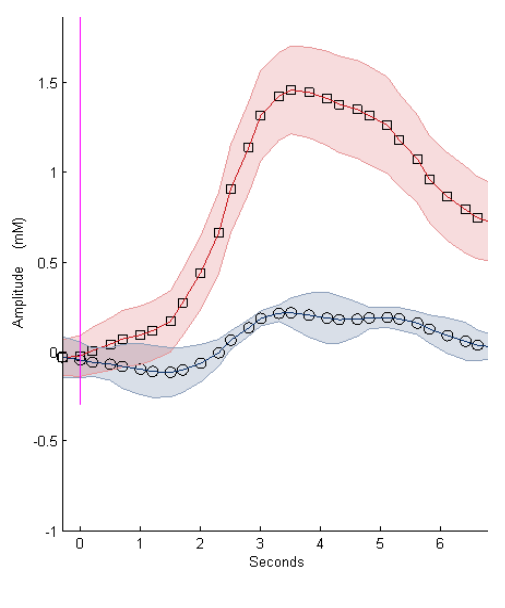

MD-DT

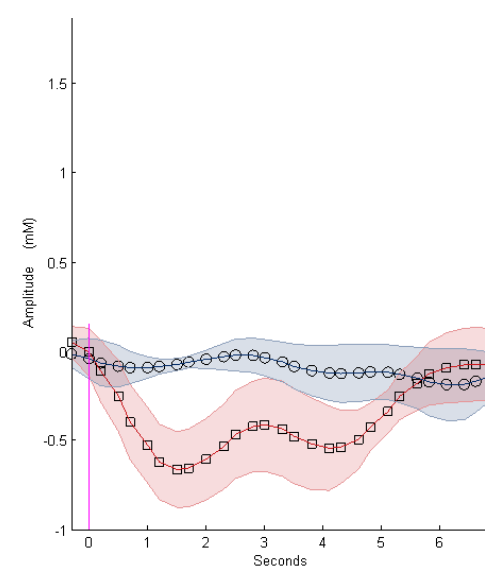

AD-DT

(b)

Figure 5: (a) Interaction between driving mode and listening for OxyHb in occipital region. ST: Single task. DT: Dual task (with listening). (b) Grand averages (including 12 participants, mean \pm standard error) hemodynamic responses from occipital region under DT according to the manual (MD) or autonomous (AD) driving condition, the inter-subject mean \pm standard error of $\mathrm{OxyHb}$ (red lines) and DeoxyHb (blue lines) are represented for the channel between $\mathrm{POz}$ and $\mathrm{Oz}$ (amplitude $\left.(\mathrm{mM}) \times 10^{-4}\right)$. 


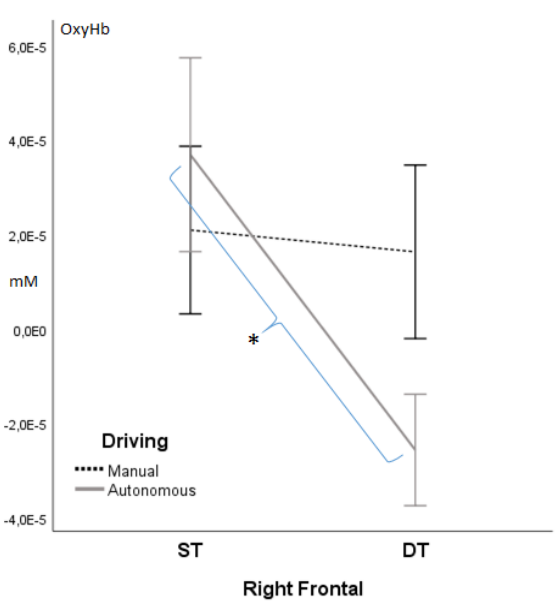

(a)

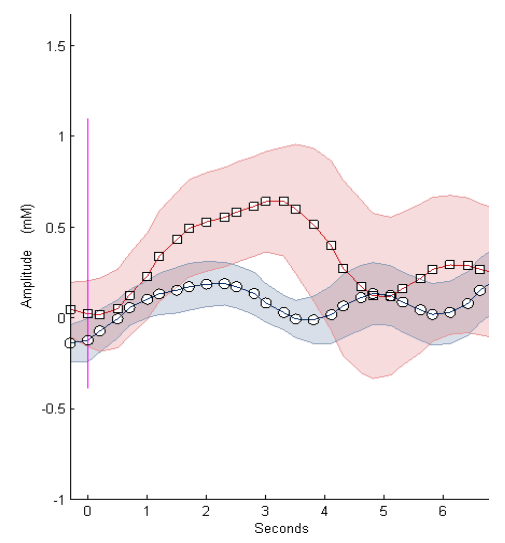

AD-ST

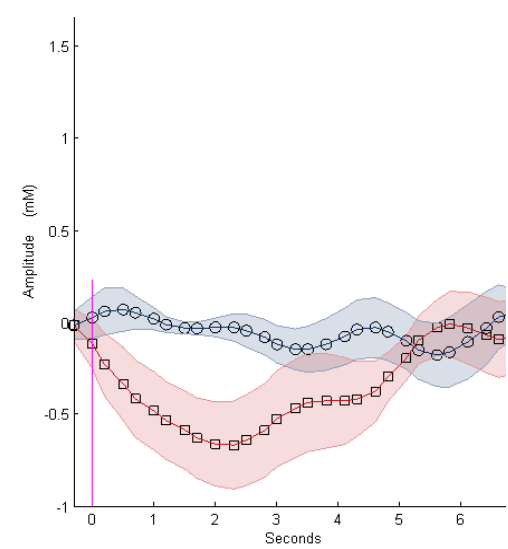

AD-DT

(b)

Figure 6: (a) Interaction between driving mode and listening for OxyHb in the right frontal region. ST: Single task. DT: Dual task (with listening). (b) Grand average (including 12 participants, mean \pm standard error) hemodynamic responses from the right frontal region in autonomous driving (AD) without (ST) and with listening (DT), and the inter-subject mean \pm standard error of OxyHb (red lines) and DeoxyHb (blue lines) are represented for the channel between AF4 and AF8 (amplitude $\left.(\mathrm{mM}) \times 10^{-4}\right)$.

Concerning DeoxyHb, an interaction between driving mode and listening was also found in the right frontal region $\left(\mathrm{F}(1,11)=4.93 ; \mathrm{p}=.048 ; \mathrm{n}_{\mathrm{p}}{ }^{2}=0.310\right)$. The post-hoc analysis showed a significant difference between manual and autonomous driving in the single task condition $(\mathrm{p}=.006)$.

\section{Discussion}

As mentioned, the present work aims to investigate the hemodynamic responses evoked to relevant visual stimuli in manual and autonomous driving in a car simulator and to analyze how the inclusion of a secondary task (attentive listening to a broadcast) modulates these hemodynamic responses in both driving situations. For these aims, the fNIRS signal was recorded from frontal, temporo-parietal and occipital areas. We conducted an event-related analysis of the brain activation following illumination of a lead vehicle brake light according to 4 conditions: single- versus dual-task combined with manual versus autonomous driving. The main results were 1) a higher score of reported mind-wandering during autonomous driving as a single task than as a dual task (when attentive listening was required), 2) higher $\mathrm{OxyHb}$ concentration for manual driving compared to autonomous driving in the bilateral temporo-parietal and occipital regions, and 3) an interaction in the activation of frontal areas is found between driving and listening conditions due to the higher activation during autonomous driving as a single task compared to autonomous driving as a dual task including attentive listening (which is not significant for manual driving).

\section{Mind-wandering in manual and autonomous driving}


During manual driving, the driver must pay attention to the stimuli present in the environment and process them to respond properly. This process should reduce the natural tendency to let the mind wander, as subjectively stated (Fig. 3). In the dual task conditions, attentional resources must be shared for the two tasks (manual driving and listening to a broadcast) leading to higher cognitive workload. This sharing may lead to a decrease in mindwandering (Zhang \& Kumada, 2017). Hence, people with a higher tendency to let their minds wander (as scored by MAAS) can have comparable performance to people with a weaker tendency to let their minds wander. By contrast, during autonomous driving, correlational analyses suggest that people who scored higher in MAAS had some free attentional resources to let their minds wander, leading to lower performance in the listening task (Sec. 3.2).

\section{Driving effect: manual vs autonomous}

As expected, higher brain activation, as measured by OxyHb concentration, were found for manual driving compared to autonomous driving (see Figs. 4 to 6 and section 3.3) in the bilateral temporo-parietal and occipital regions. This result could be related to processing visual information and spatial attention and the basal low mental workload in autonomous driving situations (Sibi et al, 2016). These cognitive processes are not needed in autonomous driving where vigilance and quick reactivity are the most important needs only when a take-over is required (Casner et al, 2016). However, contrary to Sibi et al., (2016), differences in the frontal regions were not evidenced between manual and autonomous driving for the present event-related paradigm. This difference may be due to the contribution of mind-wandering when attentional resources are not needed to drive (Berthié et al., 2015).

\section{Distraction effect: attentive listening to a broadcast}

In this study, distraction was generated with a dual-task condition consisting of attentive listening. Surprisingly, we did not manage to show a general impact of the listening task on brain activation, OxyHB or DeoxyHb, regardless of the brain areas considered. Nonetheless, the brain area linked to auditory processing has been successfully studied using fNIRS (Plichta et al., 2011). Indeed, Salo, Salmela, Salmi, Numminen \& Alho, (2017) suggest that auditory distraction can be measured in a restricted area and would elicit activity enhancements only in the auditory cortex. Arguably, it would have been expected to find significant differences in temporo-parietal regions. However, during single-task driving (where there was no broadcast), there were auditory stimuli linked to driving (motor noise), which could have hidden the listening effect of the dual task.

On the other hand, in their experimental design, Salo et al., 2017 included a stream of synchronous tones and gratings where no verbal processing was needed. If verbal information is presented, there are several factors that influence brain activation besides sensorial processing, such as how interesting the information is for the listener (Horrey, Lesch, Garabet, Simmons, Maikala, 2017) or the emotional content (Spreckelmeyer, Kutas, Urbach, Altenmüller \& Münte, 2006). Even if in the present study interest and emotional content were controlled to avoid these effects, the temporal variations in these components could have an impact on the averaged measures and hide the listening effect.

\section{Interaction of driving and attentive listening}


The significant interaction between driving and listening conditions can be explained by higher activation in dorso-lateral prefrontal areas in autonomous driving for the single task compared to the dual task. This event-related activation change can be explained by greater proneness to mind-wandering in autonomous driving (without listening) due to the monotony of this task where no driving action is needed (Berthié et al., 2015).

Higher mind-wandering would decrease frontal activation during the time segments preceding the visual cues (brake lights), which are considered the baseline for the next event. This result agrees with Durantin et al., (2015), who showed that OxyHb level faded before the target stimuli in mind-wandering situations as assessed by the sustained attention to response task (SART). Hence, OxyHb level would be consequently higher after the stimulus since it measures relative oximetric brain variations. Nevertheless, we must be cautious in drawing such conclusions because inter-stimuli intervals are short and instantaneous mind-wandering is not guaranteed. Another possible explanation, not exclusive, would be that this decreased activation in the dorso-lateral prefrontal area would be due to the distractive task, as observed by Fort el al., (2010) in a driving simulator study using MEG.

The decreased activation in frontal areas in the dual task could suggest that brake lights act as distractors that interfere with the listening task. This explanation would agree with the conclusions drawn from the study by Ozawa \& Hiraki (2017) that showed decreased $\mathrm{OxyHb}$ in the prefrontal cortex associated with distraction. In the present study, this effect is observed only in autonomous driving, which is a monotonous supervision task. By contrast, for the manual driving situation, driving is always the main task, even if auditory stimuli are broadcasting. Therefore, attention would be more focused on the visual driving environment than on the broadcast, such that event-related responses show increased $\mathrm{OxyHb}$ in the occipital area reflecting visual processing of the brake lights.

However, note that, for instance, Nosrati et al., (2016) showed an increase in prefrontal brain activation whilst performing different driving tasks, such as right and left turns (with pedestrians and traffic), in the presence of auditory distractors. This discrepancy with our results might be explained by the different attentional demands of the driving tasks used. Right and left turns are highly demanding tasks compared to our braking task (without traffic or pedestrians), which can be easily automated by the drivers. In addition, the secondary tasks used were different and may have created different attentional demands. Further studies are needed to better understand the potential interactions between the demands of the main task and the demands of the secondary task.

\section{Brain hemodynamic responses in highly automated vehicles}

The weaker activation of the right prefrontal cortex, for OxyHb, in autonomous compared to manual driving together with the higher activation in the right temporo-parietal and occipital regions in manual compared to autonomous driving while listening suggest that visual cues related to the driving environment at a high level of automation could act as distractors. The incorporation of sophisticated assistance systems on board the vehicles leads us to reconsider the definition of "driving performance" and the concept of "main" and "distractive" tasks. This reconsideration is particularly true whilst driving autonomous vehicles, when secondary tasks, or even sleeping, could be allowed depending on the automation level (Casner, Hutchins \& Norman, 2016). In this context, users might consider 
the warnings to take-over as distraction from another task different from driving. This context would be particularly relevant during the first use of an automated vehicle, as the warnings have to be learned to be associated with adequate action, and they are not processed automatically.

\section{Limitations of the study}

This study has some limitations. The inter-stimuli intervals are presumably shorter than the hemodynamic response duration, leading to a rapid event-related approach (Amaro \& Barker, 2006). Hence, a study with longer windows is desirable to better establish the response latency and to avoid response overlap. Regardless, artefacts would be present in every condition without biasing the results.

Finally, despite the power analysis yielding more than 0.8 power, the results of this pilot study were obtained using a small sample. Therefore, further studies to replicate these results are needed to reinforce their interpretation and to generalize our results. In addition, cognitive and attentional processes might be partially dependent on individual characteristics, such as age, capability or expertise, and these characteristics should be considered for each population.

\section{Conclusions}

Our results suggest that visual cues (brake lights of a lead vehicle) are differently processed during manual versus autonomous driving. Besides, during autonomous driving, listening to a broadcast influences the attentional processing of these visual cues. To our knowledge, this study is the first study that showed that visual cues from the driving environment in an autonomous vehicle can be considered distractors and measured the related brain activation using fNIRS.

In future works, the emotional content of the broadcast could be manipulated to check the influence of the limbic system on fNIRS responses to distractors. Furthermore, the computation of features representing temporal variation in longer segments of hemodynamic signals could be used as input to classifiers to determine driver distraction episodes using fNIRS.

\section{References}

Allen, M., Smallwood, J., Christensen, J., Gramm, D., Rasmussen, B., Gaden Jensen, C., \& Lutz, A. (2013). The balanced mind: the variability of task-unrelated thoughts predicts error-monitoring. Frontiers in human neuroscience, 7, 743.

Amaro Jr, E., \& Barker, G. J. (2006). Study design in fMRI: basic principles. Brain and cognition, 60(3), 220-232.

Arakawa, T., Hibi, R., \& Fujishiro, T. A. (2018). Psychophysical assessment of a driver's mental state in autonomous vehicles. In press, Transportation Research Part A: Policy and Practice. 
Baldwin, C. L., Roberts, D. M., Barragan, D., Lee, J. D., Lerner, N., \& Higgins, J. S. (2017). Detecting and quantifying mind wandering during simulated driving. Frontiers in human neuroscience, 11, 406.

Berthié, G., Lemercier, C., Paubel, P. V., Cour, M., Fort, A., Galéra, C., Lagarde, E., Gabaude, C. \& Maury, B. (2015). The restless mind while driving: drivers' thoughts behind the wheel. Accident Analysis \& Prevention, 76, 159-165.

Brown, K. W., \& Ryan, R. M. (2003). The benefits of being present: mindfulness and its role in psychological well-being. Journal of personality and social psychology, 84(4), 822.

Caird, J. K., Simmons, S. M., Wiley, K., Johnston, K. A., \& Horrey, W. J. (2018). Does talking on a cell phone, with a passenger, or dialing affect driving performance? An updated systematic review and meta-analysis of experimental studies. Human factors, 60(1), 101-133.

Casner, S. M., Hutchins, E. L., \& Norman, D. (2016). The challenges of partially automated driving. Communications of the ACM, 59(5), 70-77.

Corbetta, M.,Shulman, G. L., 2002. Control of goal-directed and stimulus-driven attention in the brain. Nat Rev Neurosci. 3, 201-215.

Durantin, G., Dehais, F., \& Delorme, A. (2015). Characterization of mind wandering using fNIRS. Frontiers in systems neuroscience, 9, 45.

Fort, A., Martin, R., Jacquet-Andrieu, A., Combe-Pangaud, C., Foliot, G., Daligault, S., \& Delpuech, C. (2010). Attentional demand and processing of relevant visual information during simulated driving: A MEG study. Brain Research, 1363, 117-127.

Horrey, W. J., Lesch, M. F., Garabet, A., Simmons, L., \& Maikala, R. (2017). Distraction and task engagement: How interesting and boring information impact driving performance and subjective and physiological responses. Applied ergonomics, 58, 342-348.

Jallais, C., Gabaude, C., \& Paire-Ficout, L. (2014). When emotions disturb the localization of road elements: Effects of anger and sadness. Transportation research part F: traffic psychology and behaviour, 23, 125-132.

Jermann, F., Billieux, J., Larøi, F., d'Argembeau, A., Bondolfi, G., Zermatten, A., \& Van der Linden, M. (2009). Mindful Attention Awareness Scale (MAAS): Psychometric properties of the French translation and exploration of its relations with emotion regulation strategies. Psychological assessment, 21(4), 506-514.

Just, M. A., Keller, T. A., \& Cynkar, J. (2008). A decrease in brain activation associated with driving when listening to someone speak. Brain research, 1205, 70-80.

Lemercier, C., Pêcher, C., Berthié, G., Valéry, B., Vidal, V., Paubel, P. V., Cour, M., Fort, A., Galéra, C., Lagarde, E. \& Maury, B. (2014). Inattention behind the wheel: How factual internal thoughts impact attentional control while driving. Safety science, 62, 279-285.

Liu, Z., Zhang, M., Xu, G., Huo, C., Tan, Q., Li, Z. \& Yuan, Q. (2017) Effective Connectivity Analysis of the Brain Network in Drivers during Actual Driving Using Near-Infrared Spectroscopy. Front. Behav. Neurosci., 31.

Morais, G. A. Z., Balardin, J. B., \& Sato, J. R. (2018). fNIRS Optodes' Location Decider (fOLD): a toolbox for probe arrangement guided by brain regions-of-interest. Scientific reports, 8(1), 3341. 
Navarro, J. (2019). A state of science on highly automated driving. Theoretical Issues in Ergonomics Science, 20(3), 366-396.

Navarro, J., Reynaud, E., and Osiurak, F. (2018). Neuroergonomics of Car Driving: A Critical Meta-Analysis of Neuroimaging Data on the Human Brain behind the Wheel. Neuroscience \& Biobehavioral Reviews 95: 464-479.

Nosrati, R., Vesely, K., Schweizer, T. A., \& Toronov, V. (2016). Event-related changes of the prefrontal cortex oxygen delivery and metabolism during driving measured by hyperspectral fNIRS. Biomedical optics express, 7(4), 1323-1335.

Ozawa, S., \& Hiraki, K. (2017). Distraction decreases prefrontal oxygenation: a NIRS study. Brain and cognition, 113, 155-163.

Plichta, M. M., Gerdes, A. B., Alpers, G. W., Harnisch, W., Brill, S., Wieser, M. J., \& Fallgatter, A. J. (2011). Auditory cortex activation is modulated by emotion: a functional near-infrared spectroscopy (fNIRS) study. Neuroimage, 55(3), 1200-1207.

Salo, E., Salmela, V., Salmi, J., Numminen, J., \& Alho, K. (2017). Brain activity associated with selective attention, divided attention and distraction. Brain research, 1664, 25-36.

Scherer, K. R. (2005). What are emotions? And how can they be measured? Social science information, 44(4), 695-729.

Sibi, S., Ayaz, H., Kuhns, D. P., Sirkin, D. M., \& Ju, W. (2016, June). Monitoring driver cognitive load using functional near infrared spectroscopy in partially autonomous cars. In IEEE Intelligent Vehicles Symposium (IV), 2016 (pp. 419-425). IEEE.

Smallwood, J., and J.W. Schooler (2015). The Science of Mind Wandering: Empirically Navigating the Stream of Consciousness. Annual Review of Psychology 66: 487-518.

Spreckelmeyer, K. N., Kutas, M., Urbach, T. P., Altenmüller, E., \& Münte, T. F. (2006). Combined perception of emotion in pictures and musical sounds. Brain research, 1070(1), 160-170.

Unni, A., Ihme, K., Jipp, M., \& Rieger, J. W. (2017). Assessing the driver's current level of working memory load with high-density functional near-infrared spectroscopy: a realistic driving simulator study. Frontiers in human neuroscience, 11, 167.

Vandenberghe, R., Gitelman, D. R., Parrish, T. B.,Mesulam, M. M., 2001. Functional specificity of superior parietal mediation of spatial shifting. Neuroimage. 14, 661-673.

Villringer, A., Planck, J., Hock, C., Schleinkofer, L., \& Dirnagl, U. (1993). Near infrared spectroscopy (NIRS): a new tool to study hemodynamic changes during activation of brain function in human adults. Neuroscience letters, 154(1-2), 101-104.

Xu, Y., Graber, H. L., \& Barbour, R. L. (2014, April). nirsLAB: a computing environment for fNIRS neuroimaging data analysis. In Biomedical Optics (pp. BM3A-1). Optical Society of America.

Xu, G., Zhang, M., Wang, Y., Liu, Z., Huo, C., Li, Z., \& Huo, M. (2017). Functional connectivity analysis of distracted drivers based on the wavelet phase coherence of functional near-infrared spectroscopy signals. PLoS one, 12(11), e0188329.

Zhang, Y., \& Kumada, T. (2017). Relationship between workload and mind-wandering in simulated driving. PloS one, 12(5), e0176962. 